\title{
TRIANGLE FUZZY TRANSFORM BASED AUTOMATIC NOISE AND COLOR IMAGE REDUCTION METHODS
}

\section{Türker TUNCER}

${ }^{* 1}$ Department of Digital Forensics Engineering, Technology Faculty, Firat University, Elazig, Turkey

*Corresponding author; E-mail: turkertuncer@ firat.edu.tr

Received: 4 September 2018; Accepted: 29 December 2018

Noise reduction and image reduction are very important research area for image processing and computer vision. Many papers have been proposed for noise and image reductions. In this paper, novel triangle fuzzy sets transform (F-transform) is proposed for color image denoising and reduction. The proposed methods consist of histogram extraction, threshold points calculation, fuzzy sets construction and fuzzy tansformation phases. Firstly, histogram of the image is extracted, maximum points of histogram are calculated, and these points are considered as threshold points. Fuzzy sets are created using threshold points. Then, F-transform is applied on the overlapping and non-overlapping blocks of the images for image denoising and reduction respectively. The main objective of the presented method are to remove random noises of the images and color image reduction with satisfactory visual quality. In order to evaluate triangle fuzzy sets based Ftransform applications, variable noise intensities and block sizes are used. Mean absolute error (MAE), peaks signal noise-to-ratio (PSNR) and penalized function (PEN) are utilized for obtaining numerical results. Numerical simulations and comprasions clearly illustare that the proposed triangle F-transform is good transformation for random noises removing and image reduction.

Key words: Triangle fuzzy transform, Image denoising, Image reduction, Image processing.

\section{Introduction}

In the literature, many transformations for instance Discrete Wavelet Transform (DWT), Discrete Cosine Transform (DCT), Fractional Fourier Transform (FrFT), Fast Fourier Transform (FFT) have been proposed for image reduction, compression, watermarking, recognition and classification [1-4]. Fuzzy transform (F-transform) is one of them and it was presented by Perfilieva in the 2006 [5]. Ftransform have been used very large area in the image processing such as image reduction, compression, 
noise reduction. Martino et al. proposed a color image reduction method using F-transform [6]. They used cosine fuzzy set to implement fuzzy transformation and they utilized color images as test images. Mean square error (MSE) and penalty function (PEN) were considered as evaluation criteria. Manchanda and Sharma presented a F-transform based image fusion method [7]. The images were divided into $\mathrm{M} \times \mathrm{N}$ size of non-overlapping blocks and F-transform was applied on each blocks. Then, the F-transform values were fused using weighted method. Chandrasekharan and M presented a Ftransform based image enhancement algorithm [8]. In their letter, they used a triangle like fuzzy set and this fuzzy set called as modified triangle fuzzy set. They compared their method to other enhancement methods and this paper proved that the F-transform is successful for contrast enhancement. Manchanda and Sharma suggested a medical image fusion method using F-transform and this F-transform was used cosine fuzzy sets [9]. To evaluate their method, MRI, CT and PET datasets were used. Gregori et al. proposed an image noise reduction method using fuzzy averaging filter with correction step [10]. They used color images for evaluation and peak signal noise-to-ratio (PSNR) was utilized as performance metric. They presented visual and numerical results to show success of the method. Martino et al. presented compressed image segmentation method. The presented compression process was implemented using F-transform and segmentation process used fast generalized fuzzy c-means (FGFCM) [11]. Martino and Sessa proposed a fragile watermarking method for tampered areas localization. This method consisted of image coding using F-transform, watermark insertion, tamper detection and localization phases. This paper illustrated that F-transform is well method for image authentication [12]. Martino and Sessa presented image compression and decompression method using discrete F-transform and they proved that using theorems [13]. Mockor and Hurtik investigated relationships between similarity relations and lattice-valued F-transform [14].

In this paper, a triangle F-transform using triangle fuzzy sets are presented. The triangle fuzzy sets are constructed by using peak points of the histogram. This F-transform is utilized as noise and color image reduction operator in this paper. The main contributions of this paper are given as below.

- In this work, a novel triangle sets based F-transform is presented for noise and image reductions.

- The proposed triangle F-transform used as image compression and noise reduction method. Triangle fuzzy sets are created using histogram of the image. Noise reduction and compression effects were shown in this paper by using two methods with variable parameters. The proposed method was also compared state of art methods. The results proved success of the proposed F-transform.

- A general F-transform algoritms is presented. In this algorithm, overlapping and nonoverlapping blocks are used for image and noise reductions respectively.

\section{The proposed F-Transform based image noise reduction method}

In this method, a novel F-transform is presented and this transform used the classical and the widely used fuzzy set which is called as triangle is used. In this method, 3 triangle fuzzy sets are utilized to calculate transformation. This method consists of histogram extraction, threshold points calculation, fuzzy sets construction, membership degree calculation, fuzzy transformation using these membership degrees and fuzzy image construction phases. The steps of the presented method are given as below.

Step 1: Divide R, G and B channels into cover image.

Step 2: Extract histogram of each channel using Algorithm 1. 
Algorithm 1. Pseudo code of the histogram extraction.

Input: Image (I) with size of $\mathrm{M} \times \mathrm{N}$

Output: Histogram of I (histo) with size of 256

1: histo=zeros(256); // a vector with size of $1 \times 256$ and all // of the element are 0 .

2: for $\mathrm{i}=1$ to $\mathrm{M}$ do

3: for $\mathrm{j}=1$ to $\mathrm{N}$ do

4: $\quad \operatorname{histo}(I(i, j))=\operatorname{histo}(I(i, j))+1$;

5: endfor

6: endfor

Step 3: Divide histogram into $N$ parts. $N>1$. N represents number of the fuzzy sets.

Step 4: Calculate threshold points using Algorithm 2.

Algorithm 2. Pseudo code of the threshold points calculation.

Input: Histogram of the image (histo) with size of 256.

Output: Threshold points (thr) with size of $\mathrm{N}$.

1: for $\mathrm{i}=1$ to $\mathrm{N}$ do

2: $\quad$ counter $=\left\lfloor\frac{256}{N}\right\rfloor$

3: $\quad\left[\mathrm{m}_{\mathrm{i}} \mathrm{t}_{\mathrm{i}}\right]=\max ($ histo $((i-1) \times$ counter $+1: i \mathrm{x}$ counter $)) ; / /$ Dividing histogram and finding maximum points

4: $\quad t h r_{i}=t_{i}+(i-1) \times$ counter; // Threshold point calculation.

5: endfor

Step 5: Calculate membership degrees of the images using threshold points.

$f(i, j, k)=\left\{\begin{array}{c}\frac{C h_{i, j}}{t h r_{k}}, C h_{i, j} \leq t h r_{k} \\ 1-\left(\frac{C h_{i, j}-t h r_{k}}{255-t h r_{k}}\right), C h_{i, j}>t h r_{k}\end{array}, i=\{1,2, \ldots, W\}, j=\{1,2, \ldots, H\}\right.$

Where $C h_{i, j}$ pixel of the channel, $t h r_{k}$ is threshold point, $\mathrm{i}$ and $\mathrm{j}$ are indices of the image, $\mathrm{W}$ is width of the image (channel), $\mathrm{H}$ represents height of the image and $\mathrm{k}$ is indice of the threshold points and $k=\{1,2, \ldots, N\}$. Eq. 1 defines triangle fuzzy sets and a sample of it is shown in Fig. 1. 


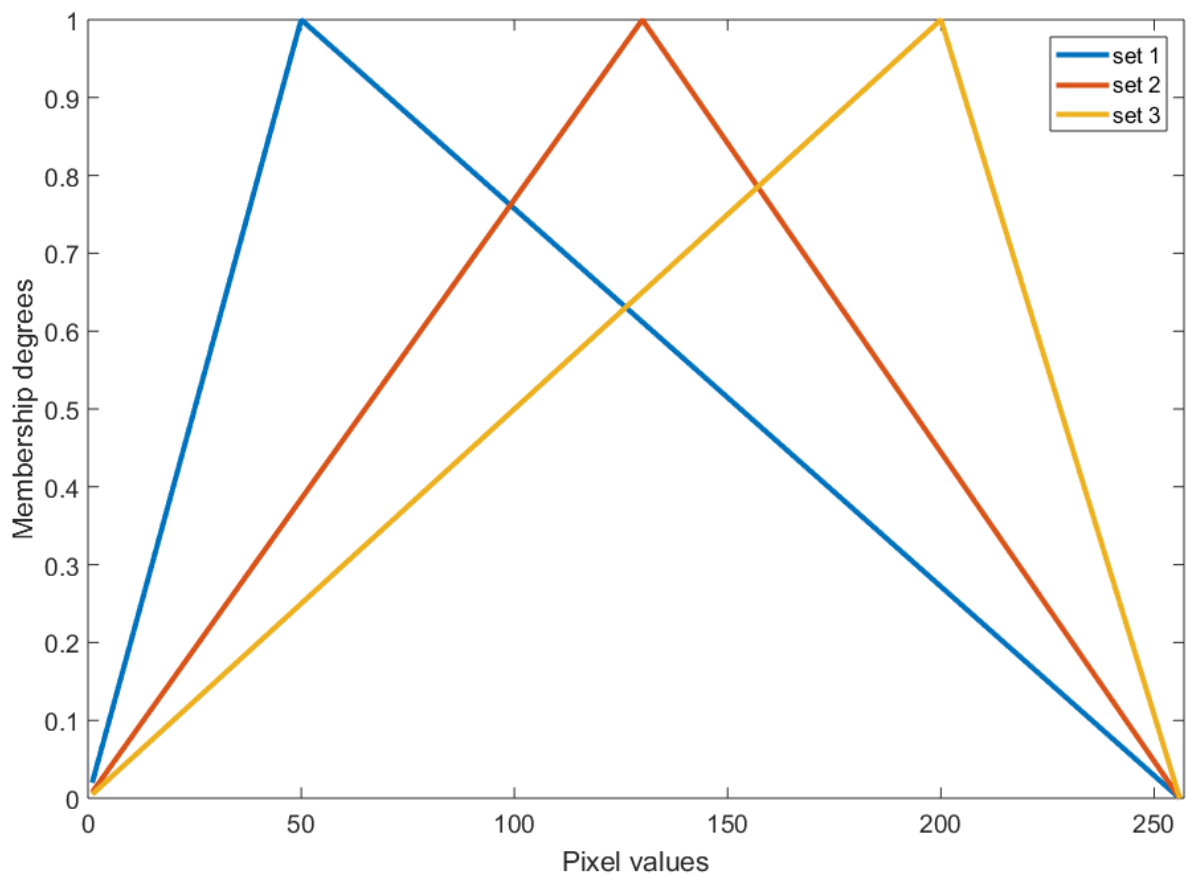

Fig. 1. The 3 triangle fuzzy sets.

Step 6: Divide image into b x b size of overlapping blocks using noise intensity.

$b=\left\{\begin{array}{c}10 I-1,10 I-1 \geq 2 \\ 2,10 I-1<2\end{array}\right.$

Step 7: Apply F-tranform to each overlapping block.

$$
\begin{aligned}
C h_{w, h}^{F} & =\frac{\sum_{i=1}^{b} \sum_{j=1}^{b} C h_{i, j} f_{i, j, 1} f_{i, j, 1} \ldots f_{i, j, N}}{\sum_{i=1}^{b} \sum_{j=1}^{b} f_{i, j, 1} f_{i, j, 1} \ldots f_{i, j, N}} \\
C h_{w, h}^{F} & =\operatorname{round}\left(C h_{w, h}^{F}\right)
\end{aligned}
$$

Step 8: Combine the $\mathrm{R}, \mathrm{G}$ and $\mathrm{B}$ channels and reconstruct fuzzy image.

\section{The proposed F-Transform based image reduction method}

In here, triangle fuzzy transform based color image reduction is presented. This method is similar to Martino et al.'s [6] method. In Ref. [6], cosine based fuzzy sets are used. In this paper, triangle based fuzzy sets are used to reduct color images. The pseudo code of the presented color image reduction algorithm is given below.

Algorithm 3. Triangle F-Transform based color image reduction

Input: Color image $(\mathrm{CI})$ with size of $\mathrm{W} x \mathrm{H}$, block size $\mathrm{b}$.

Output: Reducted Image (RI) with size of W x H. 


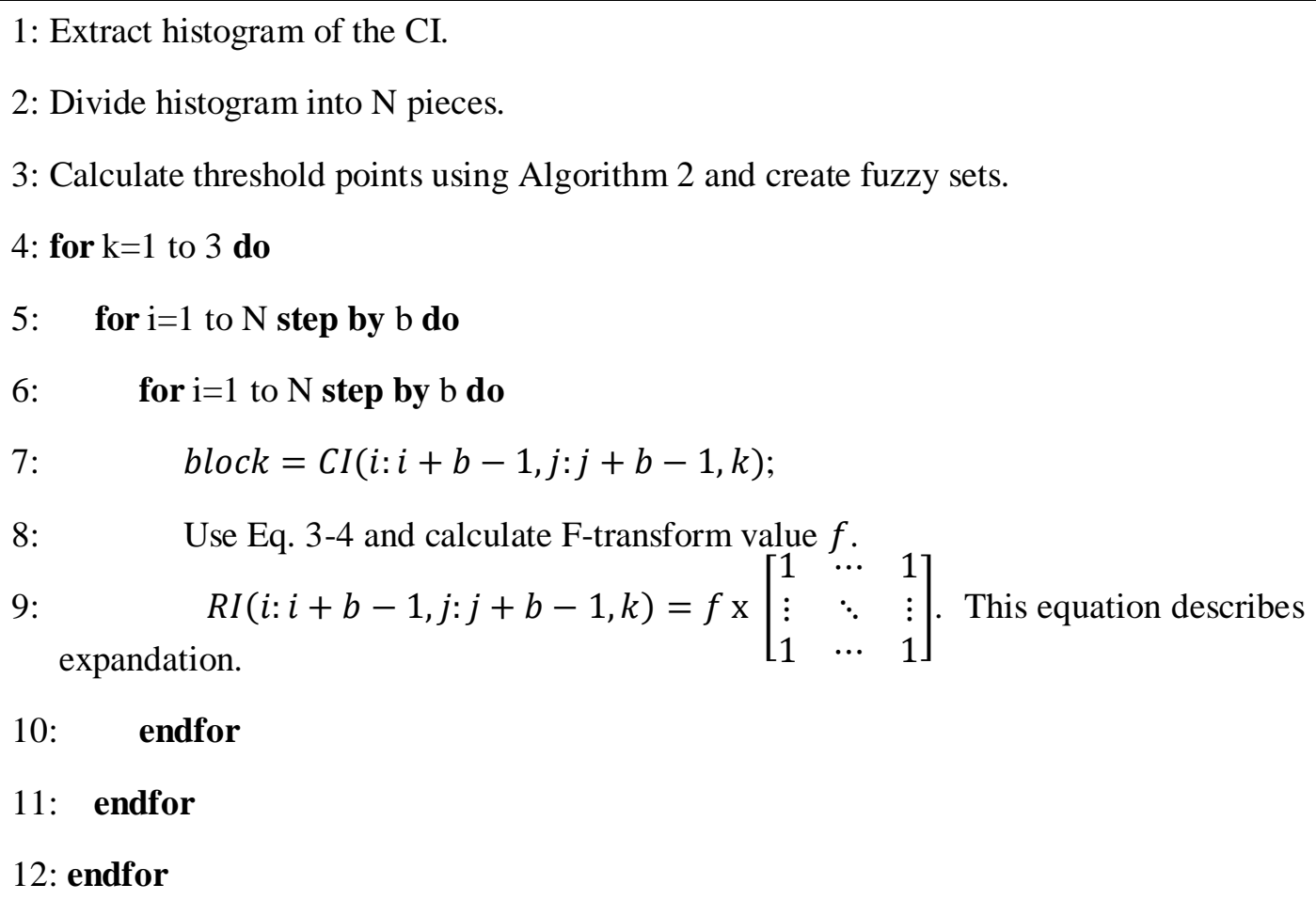

\section{Experimental Results}

In this section, numerical results of the proposed methods are obtained for performance evaluation and PSNR, MAE and Penalty function (PEN) metrics are considered to get numerical results from test images. Mathematical description of the PSNR, MAE and PEN are given Eq. 5-7. Also, 2 triangle fuzzy sets were used to obtain experiments because the best results are achieved using 2 triangle fuzzy sets.

$$
\begin{aligned}
& P S N R=10 \log _{10} \frac{255^{2} \times 3 \mathrm{WH}}{\sum_{k=1}^{3} \sum_{i=1}^{W} \sum_{j=1}^{H}\left(I_{i, j, k}-R I_{i, j, k}\right)^{2}} \\
& M A E=\frac{\sum_{k=1}^{3} \sum_{i=1}^{W} \sum_{j=1}^{H}\left|I_{i, j, k}-R I_{i, j, k}\right|}{3 W H} \\
& P E N=\frac{\sum_{i=1}^{W} \sum_{j=1}^{H} \sum_{k=1}^{3}\left|I_{i, j, k}-R I_{i, j, k}\right|^{2}}{3 W H}
\end{aligned}
$$

where $I$ is original image and $R I$ reduced image for noise or image reduction.

In order to achieve numerical results, a color image test set is used. The test images used are shown in Fig. 2. 


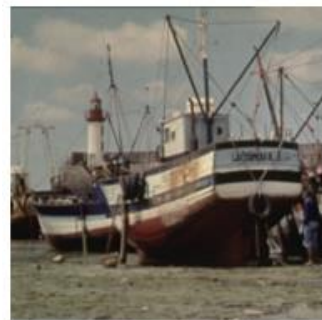

(1)

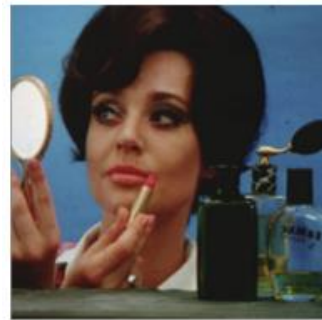

(4)

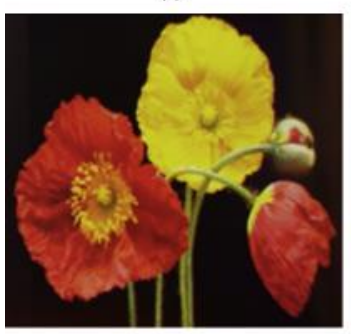

(7)

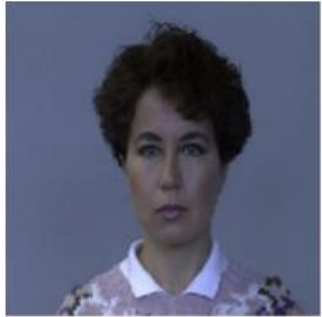

(2)

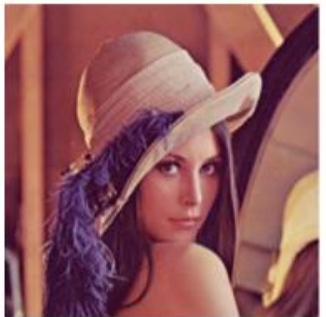

(5)

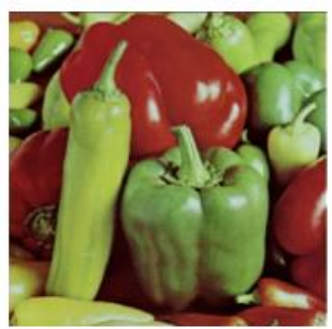

$(8)$

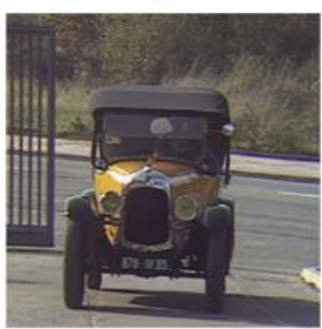

(10)

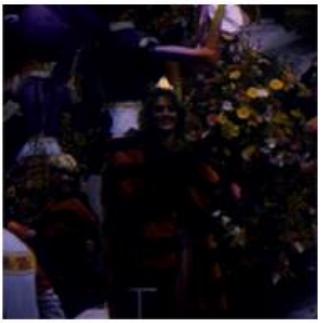

(3)

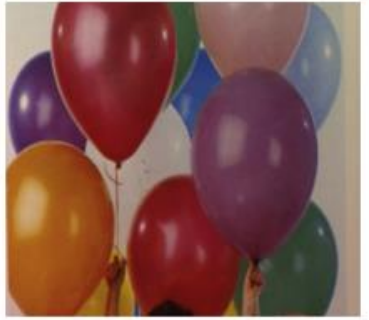

(6)

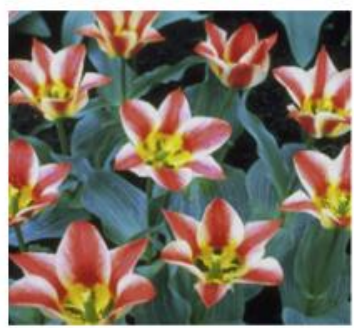

(9)

Fig. 2. Colored test images.

\subsection{Noise reduction results}

Random noises with variable intensities are used to evaluate performance of the proposed triangle based image denoising method. The simulations are implemented on the MATLAB2017a. 10 test images which are shown in Fig. 2 are used to obtain numerical results and they are listed in the Table 1. 
Table 1. Statistical results of the proposed noise reduction method with variable noise intensities.

\begin{tabular}{|c|c|c|c|c|c|c|c|c|c|c|c|c|c|c|c|c|c|c|c|c|c|c|c|c|}
\hline & \multicolumn{3}{|c|}{$10 \%$} & \multicolumn{3}{|c|}{$20 \%$} & \multicolumn{3}{|c|}{$30 \%$} & \multicolumn{3}{|c|}{$40 \%$} & \multicolumn{3}{|c|}{$50 \%$} & \multicolumn{3}{|c|}{$60 \%$} & \multicolumn{3}{|c|}{$70 \%$} & \multicolumn{3}{|c|}{$80 \%$} \\
\hline & SNR & $\mathrm{AE}$ & EN & SNR & $\mathrm{AE}$ & EN & SNR & $\mathrm{AE}$ & EN & SNR & $\mathrm{AE}$ & $\mathrm{EN}$ & SNR & $\mathrm{AE}$ & $\mathrm{EN}$ & SNR & $\mathrm{AE}$ & EN & SNR & $\mathrm{AE}$ & EN & SNR & $\mathrm{AE}$ & EN \\
\hline & 9.49 & .66 & 08.74 & 8.19 & .86 & 37.05 & 5.31 & .57 & 40.34 & 4.77 & .24 & 14.70 & 2.80 & 0.76 & 86.49 & 1.59 & 2.71 & 307.8 & 0.75 & 4.28 & 581.5 & 0.05 & 5.68 & 844.0 \\
\hline & 9.40 & .31 & 13.21 & 8.57 & .45 & 21.90 & 6.75 & .05 & 89.29 & 6.63 & .75 & 04.24 & 5.55 & .74 & 25.47 & 4.74 & .58 & 31.63 & 4.13 & .30 & 22.99 & 3.61 & .95 & 08.38 \\
\hline & 0.11 & .90 & 80.71 & 9.65 & .96 & 90.82 & 9.13 & .09 & 95.06 & 7.06 & .99 & 50.96 & 5.28 & .64 & 34.06 & 4.10 & .04 & 98.55 & 3.05 & .31 & 90.31 & 2.32 & 0.44 & 035.0 \\
\hline & 9.37 & .49 & 14.35 & 8.56 & .62 & 27.99 & 6.21 & .18 & 99.88 & 5.51 & .18 & 08.45 & 3.70 & .30 & 75.94 & 2.46 & 0.11 & 024.5 & 1.46 & 1.74 & 264.7 & 0.73 & 3.18 & 501.7 \\
\hline & 9.89 & .35 & 85.89 & 8.62 & .54 & 11.01 & 5.46 & .23 & 21.80 & 5.39 & .65 & 14.61 & 3.39 & 0.05 & 29.46 & 1.94 & 2.12 & 154.2 & 0.94 & 3.87 & 446.0 & 0.12 & 5.49 & 725.9 \\
\hline & 1.36 & .51 & 33.65 & 9.79 & .68 & 54.31 & 6.68 & .29 & 32.69 & 7.92 & .33 & 85.94 & 6.17 & .77 & 32.42 & 4.93 & .01 & 68.88 & 3.95 & .14 & 16.85 & 3.03 & .30 & 63.97 \\
\hline & 7.60 & .93 & 18.47 & 7.00 & .05 & 31.46 & 5.35 & .48 & 99.79 & 3.41 & .94 & 08.98 & 1.74 & .17 & 174.7 & 0.68 & 0.98 & 472.2 & 9.85 & 2.55 & 737.2 & 9.25 & 3.79 & 970.9 \\
\hline & 7.45 & .91 & 24.57 & 6.40 & .12 & 59.09 & 3.99 & .86 & 88.55 & 2.83 & .19 & 15.384 & 1.06 & 2.18 & 387.3 & 9.86 & 4.65 & 811.3 & 9.04 & 6.67 & 190.3 & 8.36 & 8.48 & 503.9 \\
\hline & 7.11 & .68 & 33.68 & 6.08 & .96 & 75.09 & 3.63 & .78 & 28.95 & 1.72 & 2.64 & 167.3 & 9.58 & 6.91 & 932.4 & 8.24 & 0.44 & 625.2 & 7.27 & 3.43 & 212.5 & 6.55 & 5.97 & 796.4 \\
\hline 0 & 7.32 & .42 & 47.59 & 6.67 & .59 & 61.52 & 4.86 & .22 & 48.07 & 3.63 & .01 & 99.76 & 2.07 & 1.31 & 157.0 & 1.14 & 2.93 & 436.9 & 0.49 & 4.22 & 663.1 & 9.97 & 5.35 & 864.6 \\
\hline
\end{tabular}


Visual results of samples were shown in Fig. 3 to better understand success of the proposed noise reduction method.

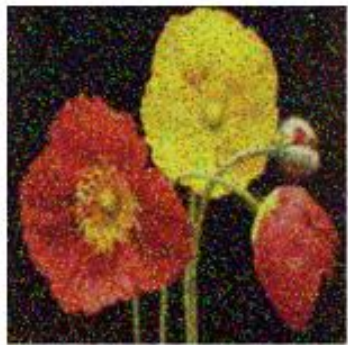

(a)

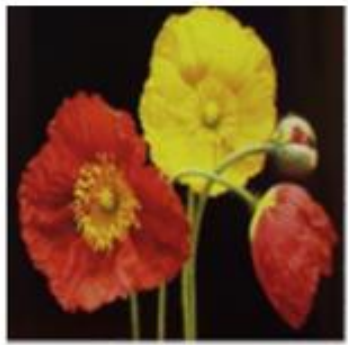

(b)

Noise intensity $=10 \%$

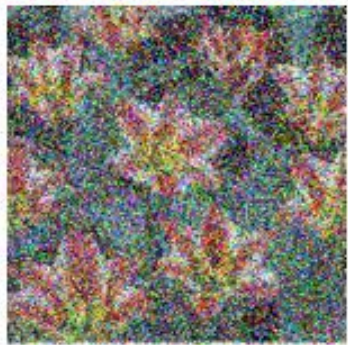

(a)

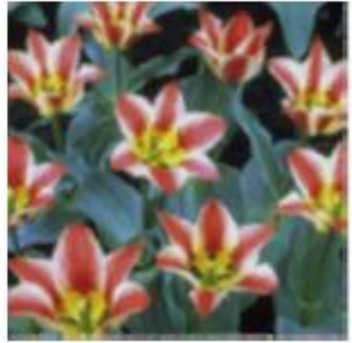

(b)

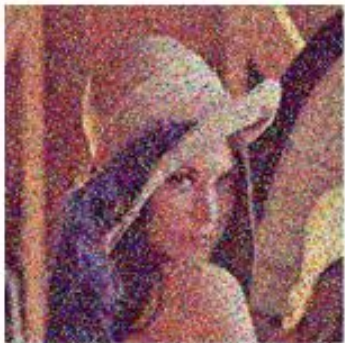

(a)

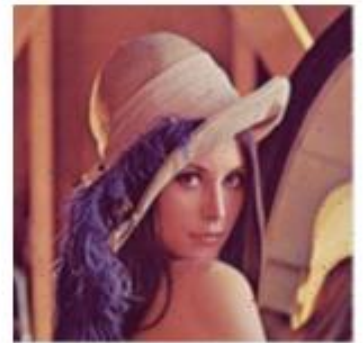

(b)

Noise intensity $=20 \%$

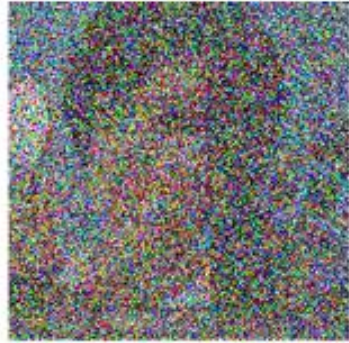

(a)

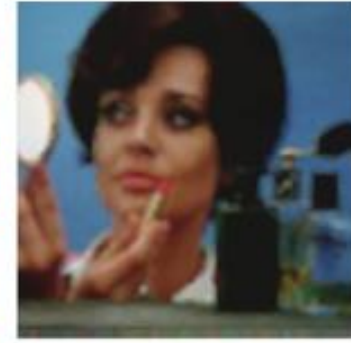

(b)

Noise intensity $=40 \%$

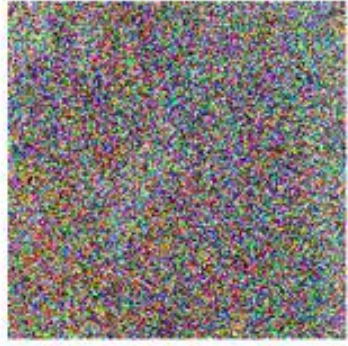

(a)

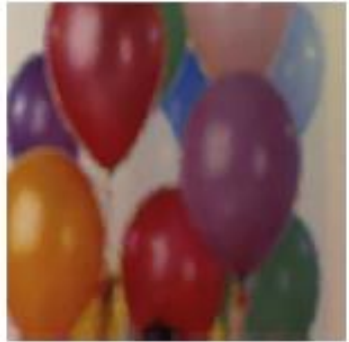

(b)

Noise intensity $=80 \%$

Fig. 3. Visual results aof the some samples (a) noisy image, (b) noise reducted image.

\subsection{Image reduction results}

$2 \times 2,3 \times 3,4 \times 4$ and $5 \times 5$ size of non-overlapping blocks are used to evaluate image reduction performance of the proposed triangle based F-transform method. MAE, PSNR and PEN values of the proposed method with these sizes of blocks were listed in Table 2.

Table 2. Results of the proposed image reduction method.

\begin{tabular}{|c|c|c|c|c|c|c|c|c|c|c|c|c|}
\hline \multirow[t]{2}{*}{ Images } & \multicolumn{3}{|l|}{$2 \times 2$} & \multicolumn{3}{|l|}{$3 \times 3$} & \multicolumn{3}{|l|}{$4 \times 4$} & \multicolumn{3}{|l|}{$5 \times 5$} \\
\hline & PSNR & MAE & PEN & PSNR & MAE & PEN & PSNR & MAE & PEN & PSNR & MAE & PEN \\
\hline 1 & 26.61 & 6.94 & 413.81 & 24.80 & 8.58 & 626.93 & 22.81 & 11.07 & 992.21 & 22.54 & 11.58 & 1502.3 \\
\hline
\end{tabular}




\begin{tabular}{|c|c|c|c|c|c|c|c|c|c|c|c|c|}
\hline 2 & 34.17 & 2.24 & 67.95 & 31.93 & 2.86 & 118.11 & 30.25 & 3.57 & 173.88 & 29.25 & 3.94 & 218.52 \\
\hline 3 & 27.88 & 4.84 & 252.35 & 26.20 & 6.27 & 386.07 & 24.13 & 8.39 & 622.16 & 23.89 & 8.85 & 666.49 \\
\hline 4 & 30.20 & 3.83 & 151.00 & 27.78 & 5.06 & 263.48 & 25.71 & 6.63 & 426.76 & 24.96 & 7.43 & 509.41 \\
\hline 5 & 27.61 & 6.04 & 298.34 & 26.11 & 7.07 & 430.83 & 24.02 & 9.29 & 696.25 & 23.58 & 9.74 & 773.27 \\
\hline 6 & 32.11 & 3.38 & 101.03 & 29.54 & 4.37 & 178.65 & 27.40 & 5.64 & 292.28 & 26.47 & 6.20 & 358.57 \\
\hline 7 & 30.34 & 3.94 & 140.35 & 27.49 & 5.23 & 265.88 & 25.56 & 6.56 & 410.76 & 24.44 & 7.31 & 524.08 \\
\hline 8 & 23.98 & 6.39 & 703.87 & 23.32 & 8.21 & 761.78 & 21.85 & 10.41 & 1161.2 & 21.31 & 11.57 & 1248.7 \\
\hline 9 & 24.74 & 9.23 & 621.73 & 23.83 & 10.08 & 796.60 & 21.65 & 13.02 & 1199.7 & 22.07 & 12.69 & 1133.6 \\
\hline 10 & 24.44 & 8.50 & 607.42 & 22.46 & 11.00 & 924.29 & 20.36 & 14.71 & 1506.9 & 19.68 & 16.24 & 1726.1 \\
\hline
\end{tabular}

In this section, $2 \times 2,3 \times 3,4 \times 4$ and $5 \times 5$ size of blocks are used to image reduction and one pixel is constructed from each block. To compare this method to others, PEN values of methods with 3 x 3 size of blocks are used and comparisons are listed in Table 3 .

Table 3. PEN values of the proposed and previously presented methods.

\begin{tabular}{|c|c|c|c|c|c|c|}
\hline Images & F-Transform & $\begin{array}{l}\text { Belikov et al.'s [15] } \\
\text { method Algorithm } \\
1\end{array}$ & $\begin{array}{l}\text { Belikov et al.'s [15] } \\
\text { method Algorithm } 2\end{array}$ & $\begin{array}{l}\text { Belikov et al.'s [15] } \\
\text { method Algorithm } 3\end{array}$ & $\begin{array}{l}\text { Martino et al.'s [6] } \\
\text { method Algorithm } 1\end{array}$ & $\begin{array}{l}\text { The proposed } \\
\text { triangle } \\
\text { transform }\end{array}$ \\
\hline 1 & 739.47 & 703.92 & 703.51 & 703.03 & 654.75 & 626.93 \\
\hline 2 & 134.49 & 128.06 & 127.93 & 127.78 & 98.69 & 118.11 \\
\hline 3 & 432.29 & 406.82 & 404.01 & 401.6 & 334.43 & 386.07 \\
\hline 4 & 312.26 & 299.04 & 297.66 & 296.54 & 253.91 & 263.48 \\
\hline 5 & 538.45 & 513.53 & 512.39 & 511.12 & 509.55 & 430.83 \\
\hline 6 & 201.86 & 191.65 & 190.91 & 190.20 & 164.20 & 178.65 \\
\hline 7 & 292.46 & 278.04 & 276.68 & 275.42 & 272.81 & 265.88 \\
\hline 8 & 808.90 & 775.64 & 774.34 & 772.98 & 639.57 & 761.78 \\
\hline 9 & 1102.79 & 1048.1 & 1044.5 & 1041.3 & 984.21 & 796.60 \\
\hline 10 & 1015.14 & 970.28 & 968.67 & 967.22 & 979.09 & 924.29 \\
\hline Mean & 557.81 & 531.50 & 530.06 & 528.71 & 482.12 & 475.26 \\
\hline
\end{tabular}




\section{Discussions}

Discussions of this study are given as below.

- The proposed noise reduction algorithm is simple as median and mean filters. It achieved very successful results for random noises and Table 1, Fig. 2 proved that this success.

- Results of the proposed image reduction method was listed in the Table 2 and comparisons were given in the Table 3 . This method was compared to 3 state of art methods using PEN values and 10 colored test images. The average PEN value of the proposed method was calculated as 475.26. It is the best value among them. Table 3 clearly demonstrated that the proposed method also achieved the best PEN values for 5 test images.

\section{Conclusions and recommandations}

In this study, a novel triangle based F-transform is proposed and its applications, which are image denoising and reduction, are presented with two novel methods. In the noise reduction method non-overlapping blocks are used. This method uses no correction steps and succesful results are achived using the proposed triangle F-transform based noise reduction method. Random noises were used to implement simulations with $10-80 \%$ noise intensities. The proposed denoising method is a basic method and it is better than mean and median filters for random noises. By using non-overlapping blocks, a novel image reduction method is presented and the comparison results have been clearly demonstrated that the proposed image reduction method is a good image decomposition method.

In the future studies, novel applications for instance perceptual hash, image watermarking, etc. methods will be proposed and novel F-transforms will be proposed using other fuzzy sets.

\section{References}

[1] Goel A., Vishwakarma V.P., Fractional DCT and DWT hybridization based efficient feature extraction for gender classification, Pattern Recognition Letters 95 (2017) 8-13.

[2] Zhang Q., Uniqueness guarantees for phase retrieval from discrete windowed fractional Fourier transform, Optik 158 (2018) 1491-1498.

[3] Strain J., Fast Fourier transforms of piecewise polynomials, Journal of Computational Physics 373 (2018) 346-369.

[4] Avci E., Tuncer T., Ertam F., Çok katmanlı görüntü steganografi, 7. Uluslararası Bilgi Güvenliği ve Kriptoloji Konferansl, 2014.

[5] Perfilieva I., Fuzzy transforms: Theory and applications, Fuzzy Sets and Systems 157 (2006) 993 1023. 
[6] Martino F.D., Hurtik P., Perfilieva I, Sessa S., A color image reduction based on fuzzy transforms, Information Sciences 266 (2014) 101-111.

[7] Manchanda M., Sharma R., Multifocus Image Fusion Based on Discrete Fuzzy Transform, IEEE WiSPNET 2017 conference, 2017, 775-779.

[8] Chandrasekharan R., S. M, Fuzzy Transform for Contrast Enhancement of Nonuniform Illumination Images, Ieee signal processing letters, vol. 25, no. 6, june 2018.

[9] Manchanda M., Sharma R., An improved multimodal medical image fusion algorithm based on fuzzy transform, Journal of Visual Communication and Image Representation 51 (2018) 76-94.

[10] Gregori V., Morillas S., Roig B., Sapena A., Fuzzy averaging filter for impulse noise reduction in colour images with a correction step, Journal of Visual Communication and Image Representation 55 (2018) 518-528.

[11] Martino F.D., Loia V., Sessa S., A segmentation method for images compressed by fuzzy transforms, Fuzzy Sets and Systems 161 (2010) 56 - 74.

[12] Martino F.D., Sessa S., Fragile watermarking tamper detection with images compressed by fuzzy transform, Information Sciences 195 (2012) 62-90.

[13] Martino F.D., Sessa S., Compression and decompression of images with discrete fuzzy transforms, Information Sciences 177 (2007) 2349-2362.

[14] Mockor J., Hurtik P., Lattice-valued F-transforms and similarity relations, Fuzzy Sets and Systems 342 (2018) 67-89.

[15] Beliakov G., Bustince H., D. Paternain, Image reductions using means of discrete product lattices, IEEE Trans. Image Process. 21 (3) (2012) 1070-1083. 